\title{
Thyroid hormone action in the absence of thyroid hormone receptor DNA-binding in vivo
}

\author{
Nobuyuki Shibusawa, ${ }^{1}$ Koshi Hashimoto, ${ }^{1}$ Amisra A. Nikrodhanond, ${ }^{1}$ \\ M. Charles Liberman, ${ }^{2}$ Meredithe L. Applebury, ${ }^{3}$ Xiao Hui Liao, ${ }^{4}$ Janet T. Robbins, ${ }^{3}$ \\ Samuel Refetoff, ${ }^{1,4}$ Ronald N. Cohen, ${ }^{1}$ and Fredric E. Wondisford ${ }^{1}$ \\ ${ }^{1}$ Department of Medicine, Pritzker School of Medicine, The University of Chicago, Chicago, Illinois, USA \\ ${ }^{2}$ Department of Otology and Laryngology, Eaton-Peabody Laboratory, and \\ ${ }^{3}$ Department of Ophthalmology, Howe Laboratory, Harvard Medical School, Boston, Massachusetts, USA \\ ${ }^{4}$ Department of Pediatrics and the J. P. Kennedy, Jr. Mental Retardation Research Center, Pritzker School of Medicine, \\ The University of Chicago, Chicago, Illinois, USA
}

\begin{abstract}
Thyroid hormone action is mediated by thyroid hormone receptors (TRs), which are members of the nuclear hormone receptor superfamily. DNA-binding is presumed to be essential for all nuclear actions of thyroid hormone. To test this hypothesis in vivo, the DNA-binding domain of TR- $\beta$ was mutated within its P-box (GS mutant) using gene targeting techniques. This mutation in vitro completely abolishes TR- $\beta$ DNA-binding, while preserving ligand $\left(T_{3}\right)$ and cofactor interactions with the receptor. Homozygous mutant (TR- $\beta$ GS/GS ) mice displayed abnormal $\mathrm{T}_{3}$ regulation of the hypothalamic-pituitary-thyroid axis and retina identical to abnormalities previously observed in TR- $\beta$ KO (TR- $\left.\beta^{-/}\right)$mice. However, TR- $\beta$ GS/GS mutant mice maintained normal hearing at certain frequencies and did not display significant outer hair cell loss, in contrast to TR- $\beta^{-/-}$mice. DNA-binding, therefore, is essential for many functions of the TR, including retinal development and negative feedback regulation by thyroid hormone of the hypothalamic-pituitary-thyroid axis. Inner ear development, although not completely normal, can occur in the absence of TR DNA-binding, suggesting that an alternative and perhaps novel thyroid hormone-signaling pathway may mediate these effects.
\end{abstract}

J. Clin. Invest. 112:588-597 (2003). doi:10.1172/JCI200318377.

\section{Introduction}

Thyroid hormones $\left(\mathrm{T}_{4}\right.$ and $\left.\mathrm{T}_{3}\right)$ are necessary for differentiation, growth, and metabolism in mammals and other lower organisms (1). Prenatal and postnatal neurologic development in humans, for example, requires a critical level of thyroid hormone. When thyroid hormone levels are low, as in congenital hypothyroidism,

Received for publication March 18, 2003, and accepted in revised form May 20, 2003.

Address correspondence to: Fredric E. Wondisford, Department of Medicine, University of Chicago, 5841 S. Maryland Avenue, MC1027, Chicago, Illinois 60637, USA.

Phone: (773) 702-9653; Fax: (773)834-0486;

E-mail: fwondisf@medicine.bsd.uchicago.edu.

Nobyuki Shibusawa's and Koshi Hashimoto's present address is:

First Department of Internal Medicine, Gunma University School of Medicine, Maebashi, Japan.

Conflict of interest: The authors have declared that no conflict of interest exists.

Nonstandard abbreviations used: thyroid hormone receptor (TR); thyroid hormone response element (TRE); thyrotropinreleasing hormone (TRH); thyroid-stimulating hormone (TSH); hypothalamic-pituitary-thyroid (H-P-T); DNA-binding domain (DBD); mouse TR- $\beta$ (mTR- $\beta$ ); low-iodine diet containing $0.15 \%$-propyl-2-thiouracil (Lo I/PTU); methimazole (MMI); assisted mechanical ventilation (AMV); auditory brainstem response (ABR); sound pressure level (SPL); distortion product otoacoustic emission (DPOAE); outer hair cell (OHC); inner hair cell (IHC); resistance to thyroid hormone (RTH); myosin heavy chain (MHC). serious defects in cerebral cortical, cerebellar, and ear development have been observed leading to mental retardation, movement disorders, and deafness $(2,3)$.

Thyroid hormone activates many genes important in neurologic development via thyroid hormone receptors (TRs) bound to specific DNA-elements (TREs). However, thyroid hormone also inhibits gene expression in the hypothalamus (thyrotropin-releasing hormone [TRH] gene) and pituitary (thyrotropin subunit genes) (thyroid-stimulating hormone [TSH]) and in this way controls thyroid hormone production from the thyroid gland (4). TR- $\beta$ is essential for negative feedback regulation of the hypothalamic-pituitary-thyroid (H-P-T) axis (5-10), but the mechanism or mechanisms of negative regulation are still incompletely understood.

On the TRH and TSH subunit genes, TR DNA-binding has also been reported to be necessary for negative regulation by thyroid hormone (11-14). In contrast to these on-DNA models of TR action, an "off-DNA" model for inhibition by the TR- $\beta$ has also been proposed. In this model, it was suggested that both the DNA-binding domain of TR- $\beta$ and direct DNA-binding of TR within the target gene were not necessary for TR-mediated transrepression (15). Evidence for a DNAbinding independent mechanism for the nuclear hormone receptor action has also been proposed for the glucocorticoid receptor (16). 
To determine which functions of the TR in vivo require DNA-binding, we generated GS125 DNAbinding domain (DBD) mutant mice. The GS125 mutant TR- $\beta$ contains a two-amino acid mutation within the P-box of the first zinc finger of the DBD. This mutant displayed very low affinities for both positive and negative TREs in gel-mobility shift assays and was completely defective in mediating both positive and negative gene regulation by thyroid hormone (17). Moreover, this unique mutant preserved $\mathrm{T}_{3}$-binding and cofactor association and was fully functional on an artificial composite response element as a ligandinducible nuclear receptor (17).

GS125 TR- $\beta$ mutant mice were studied and compared with TR- $\beta$ KO (TR- $\beta^{-/-}$) mice generated using the same targeting strategy. GS125 mutant mice demonstrate that DNA-binding is essential for negative feedback regulation of the H-P-T axis. They also displayed identical abnormalities of opsin gene expression in cone photoreceptors as those found in TR- $\beta^{-/-}$ animals. Surprisingly, the GS125 TR- $\beta$ mutant mice showed significantly less cochlear dysfunction and histopathology than the TR- $\beta^{-/-}$mice. These results indicate that a TR- $\beta$ DNA-binding-independent pathway is important in inner ear development.

\section{Methods}

Generation of GS125 TR- $\beta$ mutant knock-in mice (GS125 KI, TR- $\left.\beta^{G S / G S}\right)$ and TR- $\beta$ KO mice (TR- $\beta$ KO, TR- $\left.\beta^{-/-}\right)$. Mouse TR- $\beta$ (mTR- $\beta$ ) genomic clones were obtained by screening a 129 mouse genomic P1 phage library with a mTR- $\beta$ cDNA probe. A targeting vector was generated from an 8-kb Kpn I-Xba I fragment containing the TR- $\beta 1$ exon 3 , which encodes the first zinc finger of the DBD (Figure 1a). The GS125 mutation was introduced into exon 3 by PCR-based site-directed mutagenesis, and the ACN cassette from pACN (18) was cloned into the Bgl II site. For the TR- $\beta$ KO mice, exon 3 was replaced with the ACN cassette (Figure $1 \mathrm{~b})$. All regions of the targeting construct derived from PCR were completely sequenced.

The linearized targeting constructs were electroporated into 129 ES cells, and G418 neomycin-resistant colonies were isolated and expanded. ES cells electroporated with the targeting vector were screened by Southern blot analysis with an external probe (Figure 1c). Two of $125 \mathrm{ES}$ cell clones for GS125 KI mice and 4 of 120 ES cell clones from TR- $\beta$ KO mice were identified. Two ES clones from each construct were then injected into C57BL/ 6 blastocysts to generate chimeric mice, and chimeric mice were bred with C57BL/6 female mice. Chimeric males derived from the targeted ES cell clones transmitted the mutation through crosses with C57BL/ 6 females, and the resulting heterozygous mice were interbred. Mice heterozygous or homozygous for the targeting event were identified by Southern blot analysis (Figure 1, d and f) and PCR (Figure 1, e and g). Self-excision of the ACN cassette in the mutant allele was also confirmed.
Genotyping of tail DNA from GS125 KI animals was performed by the Southern blot analysis and PCR using a $5^{\prime}$ external primer (5'-CCGTTGTAGCATACCTGTTTTGCA-3') and a $3^{\prime}$ match primer (5'-CACTGGCACTTACCTTGCAGCCTT- $3^{\prime}$ ) for WT allele or $3^{\prime}$ mismatch primer ( $5^{\prime}$-CACTGG CACTTACCTTGCAGCTTC-3') for targeted allele. For TR- $\beta \mathrm{KO}$ animals, tail DNA genotyping was accomplished by the Southern blot analysis and PCR using a $5^{\prime} \mathrm{KO}$ primer $\left(5^{\prime}\right.$-agccgatgtcttcacctgggaagt- $\left.3^{\prime}\right)$ and a $3^{\prime} \mathrm{KO}$ primer (5'-gaaccacagcagaaaggctgt- $\left.3^{\prime}\right)$. Mice were maintained in light/ dark cycles of 12:12 hours (changes $6 \mathrm{am} / 6 \mathrm{pm}$ ) and weaned at 4 weeks of age. All mice used in these experiments were in the same mixed background strain (129/C57BL/6), and WT animals were littermate controls. All animal experiments were performed according to protocols approved by the Institutional Animal Care and Use Committee at the University of Chicago.

Western blot analysis. TR- $\beta$ protein was detected by Western blot using a mAb directed at the TR- $\beta$ C-terminus (aa 206-461, MA1-215, Affinity Bioreagents, Golden, Colorado, USA) (17). This antibody should detect all known isoforms derived from the TR- $\beta$ locus. Tissues examined include brain, pituitary, heart, lung, liver, muscle, and kidney. In data not shown, TR- $\beta 1$ and TR- $\beta 2$ proteins were also detected in appropriate tissues using $N$-terminal specific antibodies (Upstate Biotechnology, Lake Placid, New York, USA).

Serum thyroid hormone and TSH measurements. Serum thyroid hormone levels (total $\mathrm{T}_{3}$, total and free $\mathrm{T}_{4}$ ) were measured by a solid phase radioimmunoassay (ICN Pharmaceuticals, Costa Mesa, California, USA). Mouse TSH levels were measured by a specific radioimmunoassay, as described previously (9). Thyroid hormone deficiency was induced in 7-week-old male mice in each of three groups (TR- $\beta^{+/+}$, TR- $\beta^{\mathrm{GS} / \mathrm{GS}}$, and TR- $\beta^{-/-}$) with a low-iodine diet containing $0.15 \%$ 5-propyl-2-thiouracil (Lo I/PTU) and 0.05\% methimazole (MMI) in water. After 5 weeks, animals received either vehicle or daily intraperitoneal injections of a low $(0.2 \mu \mathrm{g} / 100 \mathrm{~g}$ body weight per day), medium (0.5 $\mu \mathrm{g} / 100 \mathrm{~g}$ body weight per day), or high $(1.0 \mu \mathrm{g} / 100 \mathrm{~g}$ body weight per day) dose of $\mathrm{L}_{-} \mathrm{T}_{3}$ for 5 days each. A Lo $\mathrm{I} / \mathrm{PTU}$ diet and MMI water were given throughout the $\mathrm{L}_{-} \mathrm{T}_{3}$ treatment period. Experiments were terminated 12 to16 hours after the last injection of $\mathrm{L}_{-} \mathrm{T}_{3}$.

RNA analysis. Total RNA was extracted from pooled or individual tissues by a monophasic solution of phenol and guanidine isothiocyanate (TRIzol Reagent, Life Technologies, Invitrogen, Carlsbad, California, USA). RT and PCR were carried out on $1 \mu \mathrm{g}$ of total liver RNA using assisted mechanical ventilation (AMV) RT and Tfl DNA polymerase (Access RT-PCR system, Promega, Madison, Wisconsin, USA). PCR analysis used two pairs of primers: A-1: 5'-gcactgaagaatgagcagac-3' (mTR- $\beta 1$ nucleotides 201-210); A-2: 5'-GGTTCTCCTCTATAAGCTTC-3' (mTR- $\beta$ nucleotides 930-911); B-1: 5' - 
a
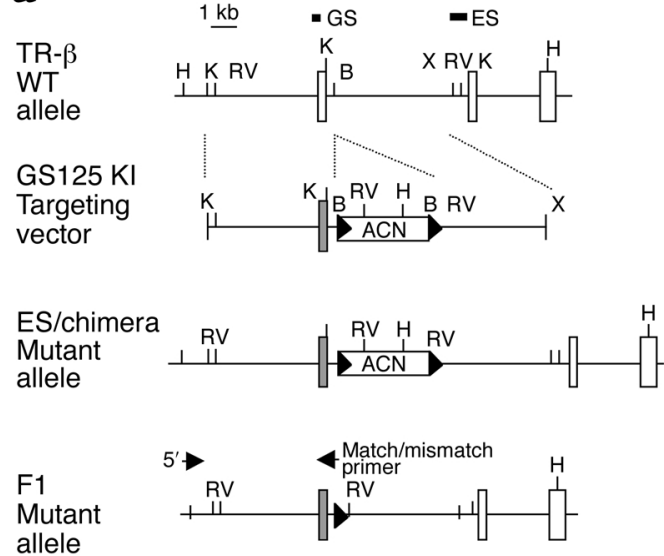

allele

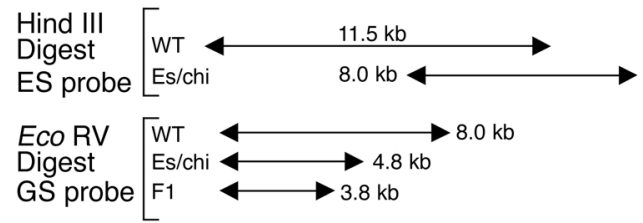

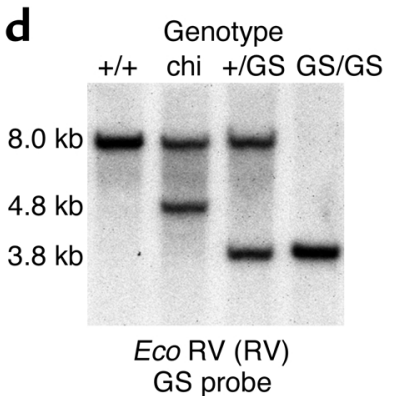

b
TR- $\beta$
WT
allele

TR- $\beta$ KO
Targeting
vector

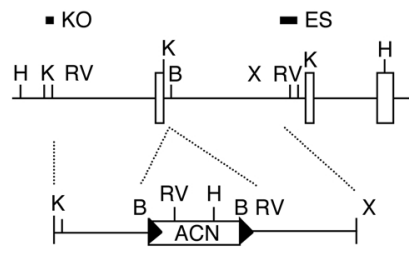

Mutant

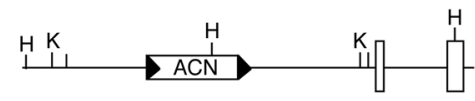

F1

Mutant

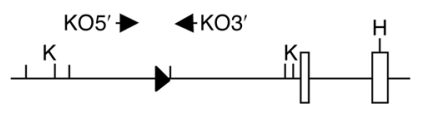

allele

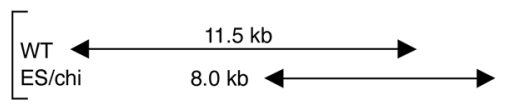

Kpn I

Digest

KO probe

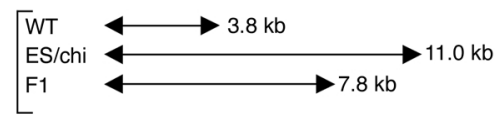

e Genotype $+/++/$ GS GS/GS

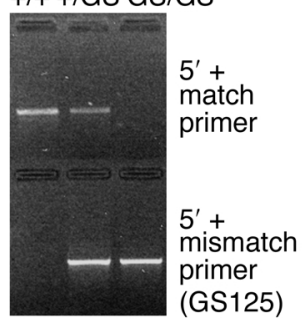

f

Genotype

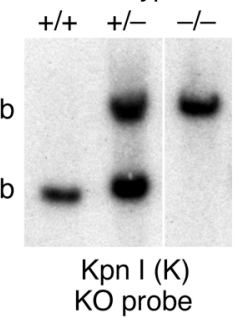

$7.8 \mathrm{~kb}$

$3.8 \mathrm{~kb}$ g Genotype $+/+$ +/- -1$-=$

TR- $\beta \mathrm{KO} 5^{\prime} / 3^{\prime}$ Intron primers GS probe

\section{Figure 1}

Generation of TR- $\beta$ GS/GS and TR- $\beta^{-/-}$mice. Schematic strategy of homologous recombination in TR- $\beta$ GS/GS $(\mathbf{a})$ and TR- $\beta^{-/-}$mice (b) is illustrated. Diagrams show the WT TR- $\beta$ locus, the targeting vectors, ES-targeted alleles, and the F1 mutant alleles after the ACN cassette is excised. The mutated exon 3 is shown as shaded boxes. H, Hind III; K, Kpn I; RV, Eco RV; B, Bgl II; X, Xba I. The locations of ES, GS, and KO probes for Southern blot analysis are indicated. The ACN cassette is flanked by loxP sites indicated by black arrowheads. Arrows indicate the positions of $\mathrm{PCR}$ primers $\left(5^{\prime}\right.$, match/mismatch, $\left.\mathrm{KO}^{\prime}, \mathrm{KO}^{\prime}\right)$ used for DNA genotyping. The site of restriction fragments obtained by Southern blot analysis is given in kb. Chi, chimeric. (c) Southern blot analysis of DNA from ES clones. WT (11.5 kb) and targeted (8.0 kb) Hind III alleles were detected by a 3' external ES probe. (d) Southern blot analysis of DNA from F2 mice resulting from a GS125 KI heterozygous intercross compared with chimeric animals. After Eco RV digestion, a 4.8-kb band was detected in targeted allele of chimeric mice using GS probe. After self-excision of ACN cassette, a 3.8-kb band is obtained from the mutant allele. (e) Genotyping of F2 KI offspring by mismatch PCR. The WT allele was detected with WT match primer set and mutant allele was detected only with the mismatch (mutant) primer set. (f) Southern blot analysis of TR- $\beta$ KO F2 siblings using the KO probe. The mutant allele demonstrated a longer ( 7.8 $\mathrm{kb}$ ) band after Kpn I digestion versus the WT allele of $3.8 \mathrm{~kb}$. (g) PCR genotyping of F2 KO mice. A 300-bp shorter band is observed in DNA from the KO versus WT allele.

AAAGCCACAGGGTACCACTA- $3^{\prime}$ (mTR- $\beta$ nucleotides 681-700); B-2: 5'-GCATTCACAATGGGTGCTTGT-3' (mTR- $\beta$ nucleotides $1117-1097$ ) to detect TR- $\beta$ transcripts. RT-PCR products from each genotype were subcloned and analyzed by cycle sequencing (PerkinElmer Applied Biosystems, Warrington, United Kingdom). For Northern blot analysis, total RNA was resolved on a $1.2 \%$ formaldehyde agarose gel, transferred onto nylon membranes, and hybridized with a radiolabeled probe as described previously (6). The hybridization bands were quantitated using NIH Image (Scion Corp., Frederick, Maryland, USA).
Histology and immunohistochemistry. Eyes were fixed overnight in $4 \%$ paraformaldehyde, processed from $5 \%$ to $30 \%$ sucrose, and frozen in embedding medium. Orientation was maintained and sections were cut at $16 \mu \mathrm{m}$, taken from the midpoint of the retina close to or at the optic nerve. Sections were double stained at $4^{\circ} \mathrm{C}$ using opsin-specific antibodies for $\mathrm{S}$ (blue) and M (green) opsin. Secondary antibodies from Molecular Probes (Eugene, Oregon, USA) were used (GAC-Alexa 488 for the M opsin, and GARAlexa 594 for the $S$ opsin). Fluorescent images were photographed using a SPOT camera (Diagnostic 
Instruments Inc., Sterling Heights, Michigan, USA), and images were printed and assembled using Adobe Photoshop (Adobe Systems Inc., San Jose, California, USA).

For cochlear analysis, ears were fixed with $2.5 \%$ glutaraldehyde and $1.5 \%$ paraformaldehyde then osmicated $\left(1 \% \mathrm{OsO}_{4}\right.$ in $\left.\mathrm{dH}_{2} \mathrm{O}\right)$ for 60 minutes, decalcified (0.1 M EDTA with $0.4 \%$ glutaradehyde) for 3 days, dehydrated in ethanol and propylene oxide, embedded in Araldite (Huntsman Advanced Materials, Salt Lake City, Utah, USA), and sectioned at 40 $\mu \mathrm{M}$. Sections were mounted in Permount (Biomedia Corp., Foster City, California, USA) and coverslipped. Photomicrographs were taken with a digital camera (C4642-95; Hamamatsu Photonics, Hamamatsu City, Japan), and processed in Adobe Photoshop. The cochlear spiral was reconstructed in $3 \mathrm{D}$, using Neurolucida software (MicroBrightField, Colchester, Vermont, USA), by tracking the heads of pillar cells. From the 3D data, the distance from the base was computed for each section through the duct, using custom software. Cochlear location was converted into frequency according to a frequency map for mice. In each section through the cochlear duct, the number of present and absent hair cells was assessed via high-powered optics (Nomarski, Edgewater, New Jersey, USA).

Auditory testing. Mice were anesthetized with xylazine $(20 \mathrm{mg} / \mathrm{kg}$ intraperitoneally) and ketamine (100 $\mathrm{mg} / \mathrm{kg}$ intraperitoneally), and a small slit was made in the pinna. Acoustic stimuli were delivered through a calibrated, closed acoustic system, consisting of two electrostatic sound sources (ES1, Tucker Davis Technologies, Alachua, Florida, USA) and one electric microphone (EK3103, Knowles Electronics, Itasca, Illinois, USA) to measure sound pressure via a probe tube near the eardrum. Auditory brainstem responses (ABRs) were measured in response to tone pips at seven logarithmically spaced test frequencies from 5.6 to $45.2 \mathrm{kHz}$. Needle electrodes were inserted at vertex and pinna, with a ground near the tail. ABR potentials were evoked with 5 -ms tone pips at 35 per second. The response was amplified, filtered, and averaged. Sound level was raised in $5 \mathrm{~dB}$ steps from $10 \mathrm{~dB}$ below threshold to $90 \mathrm{~dB}$ sound pressure level (SPL). On visual inspection of stacked waveforms, "threshold" was defined as the lowest SPL at which any wave could be detected. Distortion product otoacoustic
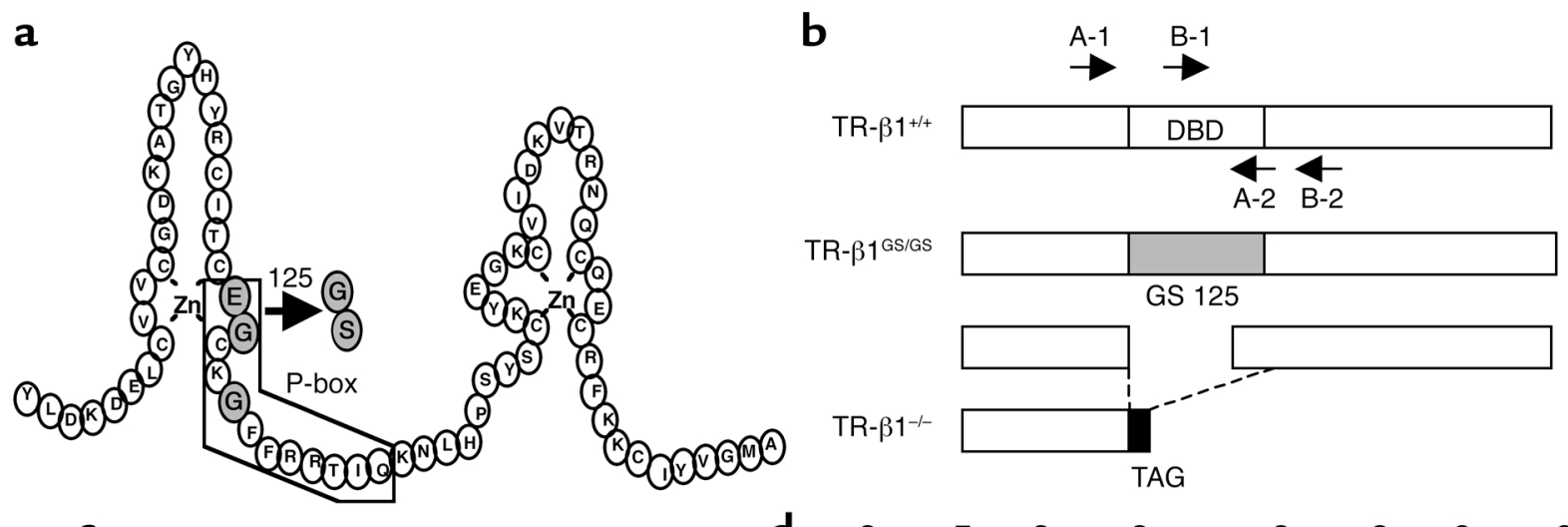

C

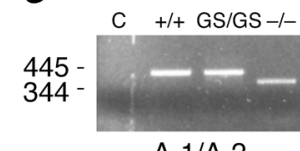

A-1/A-2

e

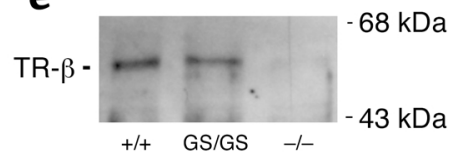

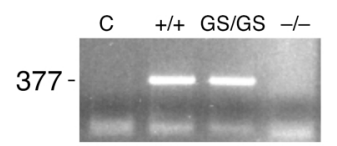

B-1/B-2

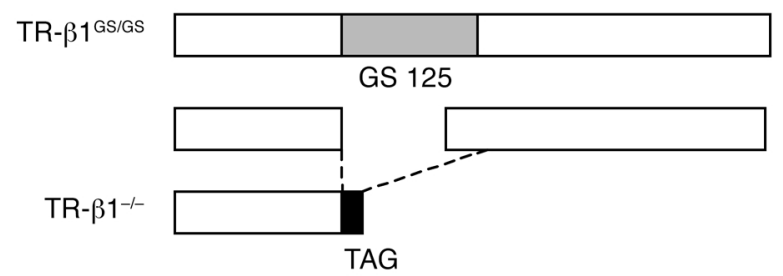

d

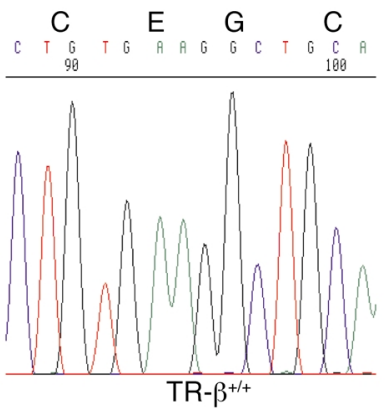

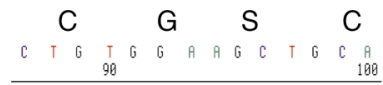

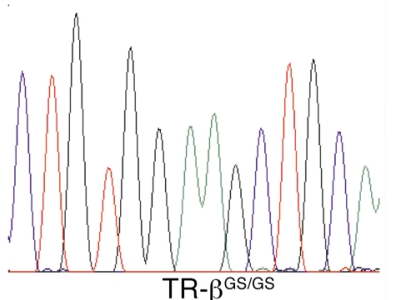

Figure 2

(a) The amino acid sequence of the DNA-binding domain of TR- $\beta$. The boxed region outlines the DNA recognition $\alpha$-helix. The shaded circles indicate the P-box amino acids. The exchange of glutamic acid 125 and glycine 126 to glycine and serine in the GS125 mutation is indicated. (b) Liver RNA was amplified by RT-PCR using primers located in sequences corresponding to exons 2 and 4 (A-1, A-2) and exons 3 and 4 (B-1, B-2), which are indicated by arrows. (c) RT-PCR results from liver RNA using the indicate primers. A 445-bp fragment from WT $(+/+)$ and TR- $\beta \mathrm{GS} / \mathrm{GS}$ animals was observed with the A primer set. A short fragment (344 bp) from the A primer set and no band from the B set were obtained from RNA transcripts from TR- $\beta^{-/-}$mice. (d) DNA sequence of the 445-bp RT-PCR fragment. The two mutated amino acids are indicated. The deleted mRNA from TR- $\beta^{-/-}$encoded a putative peptide that terminated after eight bases in exon 4 at a TAG stop codon. This was confirmed by sequencing the 344-bp fragment from TR- $\beta^{-/-}$mice (data not shown). (e) Western blot analysis of liver total cellular protein extracts from WT, TR- $\beta^{-/-}$, and TR- $\beta$ GS/GS animals (a C-terminal TR- $\beta$ monoclonal antibody was used), indicating that the GS125 mutation did not affect expression from the TR- $\beta$ locus. 


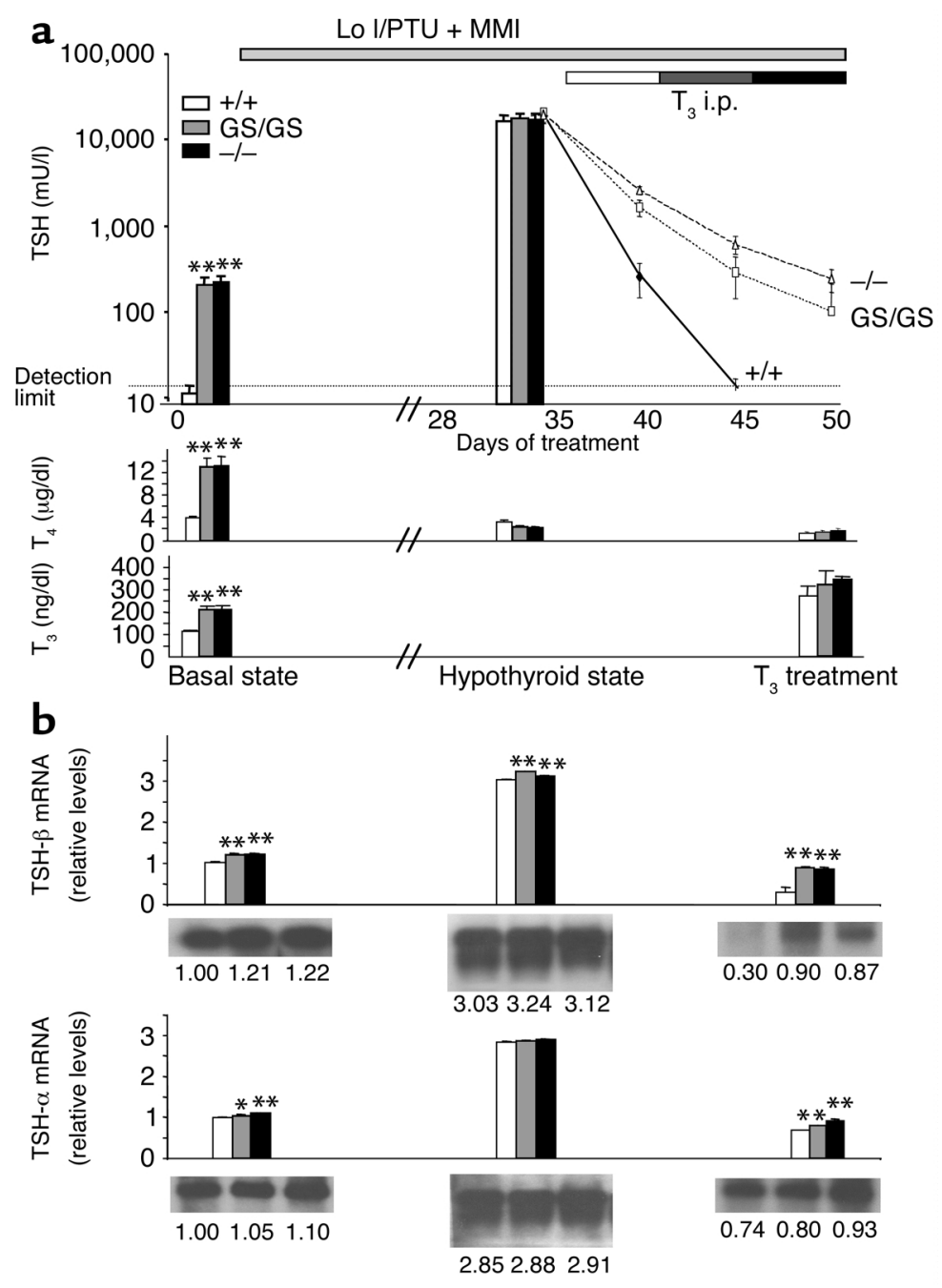

Figure 3

Effect of thyroid hormone deficiency and excess on regulation of the H-P-T axis. (a) Serum TSH levels were sequentially determined in TR- $\beta^{+/+}$(white bar, solid line), TR- $\beta{ }^{G S} / G S$ (gray bar, dotted line), and TR- $\beta^{-/-}$mice (black bar, dashed line) at the baseline, after Lo I/PTU diet+MMI water for 5 weeks, and with the additional treatment with different doses of $\mathrm{L}_{-} \mathrm{T}_{3}$ for 5 days each (top). TSH detection limit was less than $25 \mathrm{mU} / \mathrm{l}$. Serum $\mathrm{T}_{4}$ (center) and $\mathrm{T}_{3}$ (bottom) levels were also measured. Five animals were evaluated in each group, and data are shown as means \pm SEM. (b) TSH- $\beta$ (top) and common- $\alpha$ subunit (bottom) mRNA levels in the anterior pituitary of TR- $\beta^{+/+}$, TR- $\beta^{\mathrm{GS} / \mathrm{GS}}$, and TR- $\beta^{-/-}$mice using a Northern blot analysis. Each Northern blot was rehybridized with cyclophilin probe as a control, and data were normalized for each mRNA level relative to the basal state of WT animals. ${ }^{*} P<0.05,{ }^{*} P<0.01$. i.p., intraperitoneal.

emissions (DPOAEs) at $2 \mathrm{f}_{1}-\mathrm{f}_{2}$ were evoked by pairs of primary tones. Seven pairs of primary tones were tested, with $f_{2}$ values set to the same logarithmically spaced values used for ABR testing $(5.6-45.2 \mathrm{kHz})$. At each $f_{2}$ value, sound pressure of the primaries was raised in $5 \mathrm{~dB}$ steps from 15 to $80 \mathrm{~dB}$ SPL. A fast Fourier transform was computed on the averaged ear canal sound pressure waveform, and the entire process was repeated five times. The resultant five spectra were averaged to produce the final spectrum, from which $2 \mathrm{f}_{1}-\mathrm{f}_{2}$ DPOAE amplitude and surrounding noise floor were extracted.
Statistical analysis. Values are reported as mean \pm SEM. Data were analyzed by ANOVA and Student $t$ test.

\section{Results}

To address the importance of TR- $\beta$ DNA-binding for receptor function in vivo, the GS125 mutation was introduced into the TR- $\beta$ locus (exon 3, Figure 2a) via homologous recombination in $E S$ cells. The amino acids substituted for those in TR- $\beta$ are normally found in the glucocorticoid and related members of the receptor superfamily. To generate control TR- $\beta^{-/-}$animals, a second targeting vector was designed by deleting exon 3 . Both targeting vectors incorporated a self-excising neo cassette (ACN cassette) (18). Homologous recombination in ES clones was identified by Southern blot analysis and PCR (Figure 1). Chimeric males were derived from two targeted ES cell clones from each of the vectors and transmitted the mutation through crosses with C57BL/ 6 females; heterozygous mutant mice were interbred.

To establish that TR- $\beta$ protein was deleted or mutated in mice, we first examined TR- $\beta$ transcripts using RT-PCR performed on total RNA extracted from adult mouse liver tissue of WT (TR- $\left.\beta^{+/+}\right)$, GS125 mutant homozygous (TR- $\beta$ GS/GS), and TR- $\beta$ KO homozygous (TR- $\beta^{-/}$) animals (Figure $2 \mathrm{~b}$ ). With the use of primers located in TR- $\beta$ exon 2 and 4 (A-1 and A-2) and exon 3 and 4 (B-1 and B-2), PCR products from TR- $\beta$ GS/GS mice were the same size as detected from the WT animals (Figure 2c); the presence of GS125 mutation was confirmed by subsequent sequencing (Figure 2d). The transcript from TR- $\beta^{-/-}$mice obtained with A primer set was approximately $100 \mathrm{bp}$ shorter than the WT product, and no transcript was detected with $B$ primer set, indicating that these animals carried the exon 3 deletion. As reported previously, deletion of exon 3 sequences results in an aberrant open reading frame, as exon 2 is fused to exon 4 (5). Thus, the translated protein produced from the TR- $\beta^{-/-}$locus would lack the DNA-binding and ligand-binding domains. It has been reported the the TR- $\beta$ gene generates four TR- $\beta$ transcripts variants - TR- $\beta 1$, TR- $\beta 2$, TR- $\beta 3$, and TR- $\Delta \beta 3$ - using different promoters and alternative splicing. DNA binding should be disrupted in all transcripts by this alteration except in TR- $\Delta \beta 3$, where this transcript already lacks the exon 3 peptide sequence because of the use of a downstream translational start codon (19). Similarly, the GS125 mutant would be 
present in all three functional TR- $\beta$ isoforms (TR- $\beta 1$, TR- $\beta 2$, and TR- $\beta 3$ ). GS125 mutant TR- $\beta$ protein was expressed at the same level as WT TR- $\beta$ protein, as indicated by Western blot analysis of liver (Figure 2e) and pituitary (data not shown) extracts, indicating that the mutation did not affect expression from the TR- $\beta$ locus. Despite multiple attempts, we have been unable to detect TR- $\Delta \beta 3$ protein by Western blot analysis in any tissues we have examined (see Methods, data not shown). TR- $\beta^{\mathrm{GS} / \mathrm{GS}}$ and TR- $\beta^{-/-}$mice were born with no gross anatomic or functional abnormalities and were viable through adulthood. Both males and females from each genotype displayed normal fertility.

The synthesis and secretion of thyroid hormones are regulated by negative-feedback control of the H-P-T axis. To elucidate the requirement of DNAbinding by TR- $\beta$ in negative transcriptional regulation, we evaluated the axis in TR- $\beta^{\mathrm{GS} / \mathrm{GS}}$ animals. Total $T_{3}$ and total $T_{4}$ levels were significantly elevated (two- to threefold) in TR- $\beta$ GS/GS mice compared with WT mice (Figure $3 \mathrm{a}$ ). Free $\mathrm{T}_{4}$ levels were also elevated (twofold) in TR- $\beta$ GS/GS mice (data not shown). Similar results were obtained in both male and female mice. Basal serum TSH in TR- $\beta$ GS/GS mice was significantly elevated (20-fold) compared with WT animals and similar to results obtained in TR- $\beta^{-/-}$mice (Figure 3a). Serum TSH levels were not suppressed despite high-circulating thyroid hormone concentrations, indicating that TR- $\beta$ GS/GS mice have central thyroid hormone resistance - at the level of the pituitary and hypothalamus. In support of this finding, the number of anterior pituitary cells, which stained for TSH- $\beta$ subunit on immunohistochemical analysis, was also increased in both TR- $\beta^{\mathrm{GS} / \mathrm{GS}}$ and TR- $\beta^{-/-}$mice by 1.3 -fold compared with control animals (data not shown). Histologic comparison of the thyroid in TR- $\beta^{-/-}$and TR- $\beta$ GS/GS mice demonstrated an overall enlargement of gland as predicted from the serum hormone levels (data not shown) and in agreement with observations from a previous report (5).

To investigate further the molecular mechanism of abnormal regulation of the H-P-T axis in TR- $\beta$ GS/GS mice, mice were examined in both a thyroid hormone-deficient and an excess state. After 5 weeks of a Lo I/ PTU diet supplemented with MMI in the water, TSH values were markedly elevated in all groups. $\mathrm{L}-\mathrm{T}_{3}$ treatment was then begun using sequential daily intraperitoneal injections of $\mathrm{L}^{-} \mathrm{T}_{3}$ at three different concentrations. Unlike in control animals, serum TSH and TSH subunit mRNA levels (Figure $3 \mathrm{~b})$ in $\mathrm{TR}-\beta^{\mathrm{GS} / \mathrm{GS}}$ and TR- $\beta^{-/-}$mice were not suppressed completely even at the highest $\mathrm{T}_{3}$ dose, indicating markedly reduced sensitivity to negative feedback regulation by thyroid hormone. These data demonstrate that regulation of the H-P-T axis is equally impaired in GS125 mutant and TR- $\beta$ KO mice, indicating that TR- $\beta$ DNA-binding is essential for negative feedback regulation of the H-P-T in vivo.
One of the TR- $\beta$ isoforms, TR- $\beta 2$, has a restricted expression pattern in mammals and includes the retina, inner ear, and pituitary gland $(20,21)$. Homozygous deletion of TR- $\beta 2$ in mice resulted in a lack of $M$ opsin expression in the retina - found in green cones - and a redistribution of S opsin expression - found in blue cones (7). Cone opsin distribution in the retinas of TR- $\beta$ GS/GS and TR- $\beta^{-/-}$mice was therefore evaluated using immunohistochemistry and opsin-specific antibodies. In WT mouse retina, $\mathrm{S}$ cones were found in the ventral retina and were significantly less abundant in the dorsal retina; in contrast, $\mathrm{M}$ opsin is more highly expressed in the dorsal retina compared with the ventral retina (Figure 4) (22). TR- $\beta$ GS/GS and TR $-\beta{ }^{-/-}$mice, however, have no expression of $\mathrm{M}$ opsin throughout their retina. Also, TR- $\beta^{\mathrm{GS} / \mathrm{GS}}$ and TR- $\beta^{-/-}$ mice showed significantly more $S$ opsin expression in the dorsal cones compared with WT mice, and a gradient of expression was not apparent. These changes

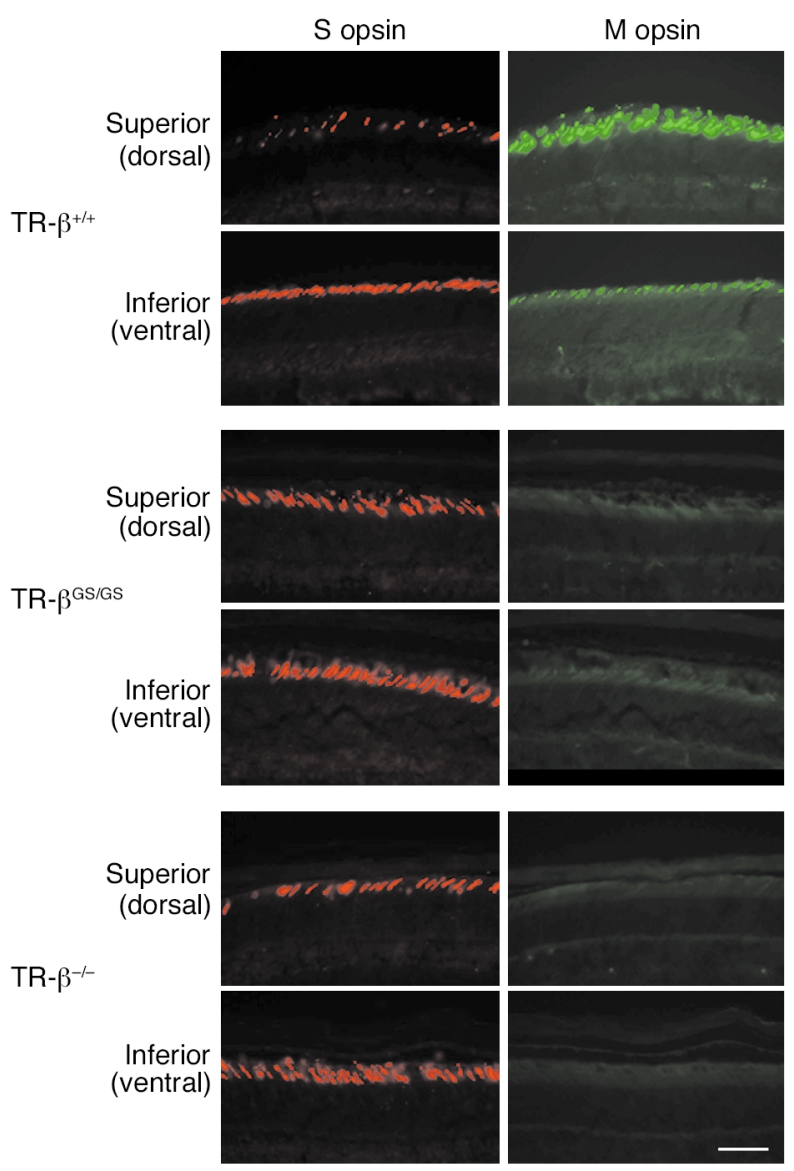

\section{Figure 4}

Selective loss of M-cone and altered S-cone distribution in TR- $\beta$ GS/GS and TR- $\beta^{-/-}$mutant mice. The left panels show $S$ opsin immunostaining visualized with red fluorescence. The right panels display $M$ opsin-specific immunostaining labeled with green fluorescence in retina of TR- $\beta^{+/+}$, TR- $\beta$ GS/GS, and TR- $\beta^{-/-}$mice. Both superior (dorsal) and inferior (ventral) regions are displayed from each genotype. TR- $\beta$ GS/GS and TR- $\beta^{-/-}$mice showed S opsin expression in significantly more dorsal regions of cone photoreceptors compared with TR- $\beta^{+/+}$mice and had an absence of $\mathrm{M}$ opsin expression throughout the retina. 


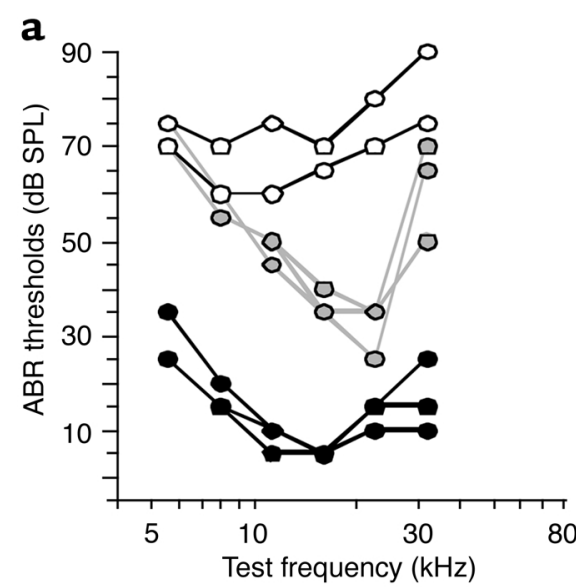

C
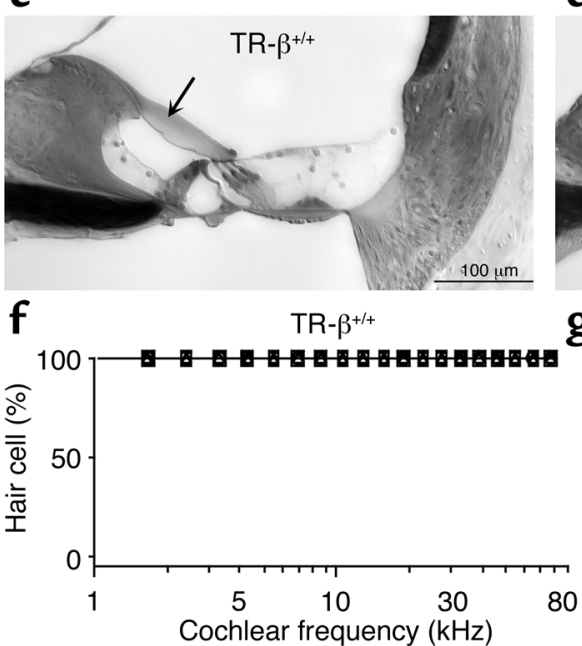

b

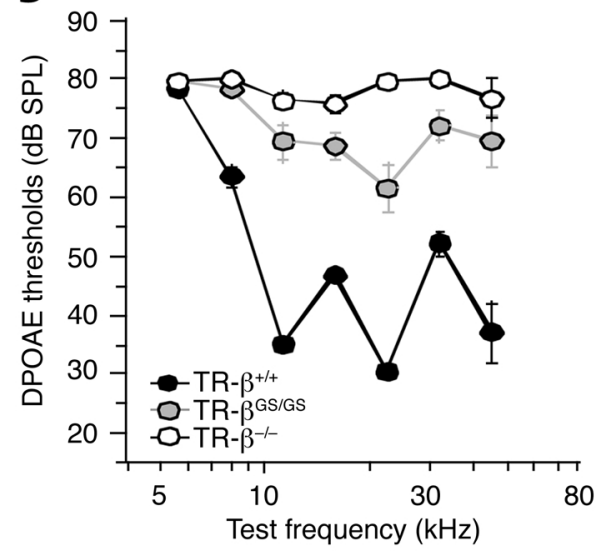

d

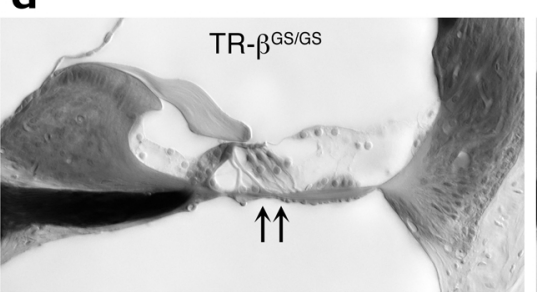

g

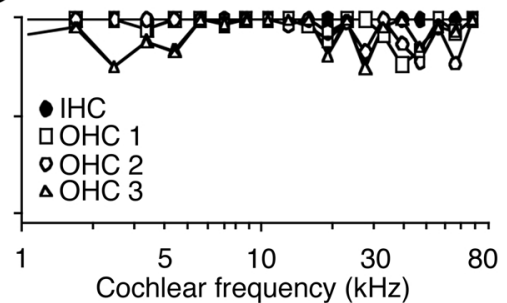

e

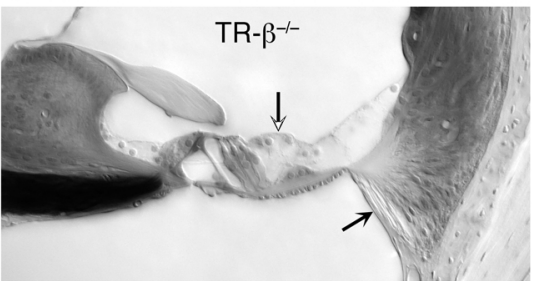

h TR- $\beta^{-1-}$

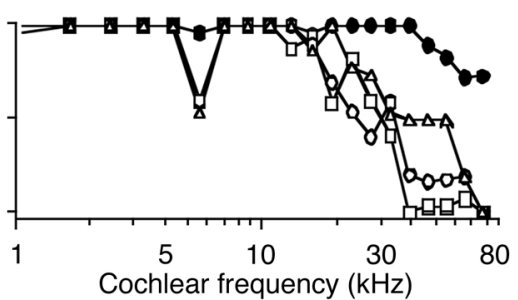

Figure 5

Cochlear function and histopathology in TR- $\beta$ mutant animals. (a) ABR thresholds for TR- $\beta$ GS/GS and TR- $\beta \beta^{-/-}$mice are elevated with respect to TR- $\beta^{+/+}$, but loss is greater in TR- $\beta^{-/}$. Data from individual 8 -week-old animals are shown. (b) DPOAE thresholds for TR- $\beta$ GS/GS and TR- $\beta^{-/-}$mice are elevated with respect to WT; however, TR- $\beta^{-/-}$exhibited a much greater deficit. Data are mean \pm SEM. Group sizes were 10,18 , and 14 for TR- $\beta^{+/+}$, TR- $\beta^{\mathrm{GS} / \mathrm{GS}}$, and TR- $\beta^{-/-}$, respectively. (c-e) Photomicrographs of the upper basal turn (cochlear frequency approximately $16 \mathrm{kHz}$ ) from WT and TR- $\beta$ mutant cochleas. Arrows indicate: (c) tectorial membrane in TR- $\beta^{+/+} ;(\mathbf{d})$ misaligned feet of outer pillar cells in TR- $\beta^{\mathrm{GS} / \mathrm{GS}}$; (e) collapse of outer supporting cells (unfilled) and loss of spiral ligament fibrocytes in TR- $\beta^{-/-}$( filled). Scale bar in $\mathbf{c}$ applies to all three images. (f-h) Basal-turn OHC loss is seen in TR- $\beta^{-/-}$mice. Data from one ear of each genotype are shown. Symbol key in $\mathbf{g}$ applies to all three panels.

are similar to those observed in TR- $\beta 2 \mathrm{KO}$ mice, indicating that the DNA-binding function of TR- $\beta$ was essential for cone opsin gene expression in the retina. Like the retina, thyroid hormone is also crucial for inner ear development. Thus, cochlear function in TR- $\beta$ mutants was assessed using two methods: (1) ABR, which represents the summed sound-evoked electrical activity of neurons in the auditory nuclei; and (2) DPOAE, which measures acoustic signals in the ear canal on presentation of two continuous tones $(23,24)$. As such, DPOAEs are a sensitive index of normal outer hair cell (OHC) function (25) and are not affected by destruction of inner hair cells (IHCs) and/or the cochlear neurons that innervate them.

ABR data (Figure 5a) showed profound hearing losses in the TR- $\beta^{-/-}$mice, with threshold elevations of approximately $60 \mathrm{~dB}$ at all test frequencies. Cochlear dysfunction was significantly less in TR- $\beta \mathrm{GS} / \mathrm{GS}$ mice, where threshold elevations of only approximately 20 to $30 \mathrm{~dB}$ were seen at some test frequencies and hearing was better than TR- $\beta^{-/-}$mice at all frequencies except $5 \mathrm{kHz}$. Although DPOAE thresholds were elevated in both TR- $\beta$ mutants, threshold elevations were significantly greater in TR- $\beta^{-/-}$versus TR- $\beta^{\mathrm{GS} / \mathrm{GS}}$ mice (Figure 5b). The DPOAE test also underestimated the degree of threshold shift for the receptor mutants because many TR- $\beta^{-/-}$ears (7 of 14$)$ showed no DPOAE responses even at the highest SPLs, whereas none ( 0 of 18) of the TR- $\beta \mathrm{GS} / \mathrm{GS}$ ears were nonresponsive.

Cochlear histology was also assessed using lightmicroscopic examination of osmium-stained, plastic sections. A number of histopathologies were observed in both TR- $\beta$ mutant lines. First, throughout the cochlea, the cross-sectional area of the tectorial 
membrane (arrow in Figure 5c) was smaller in WT versus mutant animals, and an overall abnormal shape was reproducibly present in the mutant animals. Second, in the apical half of the cochlea, both TR- $\beta^{-/-}$and TR- $\beta^{\mathrm{GS} / \mathrm{GS}}$ mice showed irregularities in the position of the outer pillar feet (arrows in Figure $5 \mathrm{~d}$ ), which was never seen in WT animals. Other histopathologies were uniquely observed in TR- $\beta^{-/-}$ ears including: (1) fibrocyte degeneration in the spiral ligament in basal half of the cochlea (filled arrowhead, Figure 5e) and (2) collapse of the outermost supporting cells (open arrowhead, Figure 5e).

An unexpected and striking histopathologic difference was found, however, when the number of hair cells throughout the cochlea was quantitated. As shown in Figure 5h, there was a significant loss of OHCs in the basal third of the cochlea in the TR- $\beta^{-/-}$ mice. In some areas, corresponding to the highest frequency hearing regions, no OHCs could be found. In contrast, TR- $\beta^{\mathrm{GS} / \mathrm{GS}}$ mice had nonsignificant and scattered OHC loss throughout the basal third of the cochlea (Figure $5 \mathrm{~g}$ ). OHCs were found in all regions of the cochlea and no area had less than $70 \%$. Preservation of OHCs in these animals is consistent with the measurable DPOAE responses (Figure $5 \mathrm{~b}$ ).

\section{Discussion}

There are two distinct TR genes, TR- $\alpha$ and $T R-\beta$, and each gene has several alternative spliced products, including the predominant forms, TR- $\alpha 1$, TR- $\beta 1$, and TR- $\beta 2$ (26). Essentially all tissues contain TRs, but there are important differences in the concentration of specific isoforms in individual tissues. Of the TR- $\beta$ isoforms, TR- $\beta 1$ is most widely expressed and particularly abundant in the liver and kidney, but it is also present in the pituitary, hypothalamus, and the developing nervous system. The expression of TR- $\beta 2$, in contrast, is largely restricted to the pituitary, hypothalamus, retina, and the developing inner ear. Accordingly, it has been described in several mouse models that TR- $\beta$ isoforms have an essential role in gene regulation within the H-P-T axis, inner ear, and retina (5-8).

We have generated TR- $\beta$ KI mice expressing a P-box mutation within the DNA-binding domain that severely impaired its ability to bind to DNA (17). The mutant TR- $\beta$ GS/GS mice displayed elevated serum levels of thyroid hormones and a hyperplastic thyroid gland caused by inappropriate secretion of TSH from the anterior pituitary. This effect on the H-P-T axis was almost identical to that found in mice lacking TR- $\beta$ (TR- $\beta^{-/}$). After induction of hypothyroidism, treatment of WT mice with graded dose of $\mathrm{T}_{3}$ rapidly and completely suppressed both serum TSH and TSH subunit mRNA levels (Figure 3). In contrast, responses to $T_{3}$ treatment were blunted in the TR- $\beta \beta^{\mathrm{GS}} / \mathrm{GS}$ and TR- $\beta^{-/-}$mice, explaining the cause of thyroid hormone excess in these animals. We have previously reported that the GS125 mutation disrupted
TR- $\beta$ binding to both positive and negative TREs, while preserving normal interactions with both corepressors and coactivators (17). Interaction with the AP-1 transcription factor should also be normal, as these interactions occur either directly or indirectly (via coactivators) with the TR- $\beta$ C-terminal domain $(27,28)$. In data not shown, moreover, the mutant TR- $\beta$ was as effective as the WT TR- $\beta$ in inhibiting an AP-1 reporter construct in transfection studies. This study now clearly demonstrates that DNA binding of TR- $\beta$ is essential for thyroid hormone negative feedback regulation of the H-P-T axis in vivo.

The molecular basis of resistance to thyroid hormone (RTH) has been demonstrated to be due to mutations of the TR- $\beta$ gene. With the exception of the initial RTH family described in 1967 that had a deletion of the TR- $\beta$ allele, all TR- $\beta$ mutations causing RTH have been found in the hinge and ligand-binding domains (29). No RTH point mutations have been so far reported in the DBD of TR- $\beta$. GS125 KI homozygous mice (TR- $\beta$ GS/GS) were viable and fertile and had hyperthyroidism as observed in the RTH patients; heterozygous mice (TR- $\beta^{+/ G S}$ ) had normal thyroid function, indicating that the mutant TR- $\beta$ lacked dominant negative activity. The GS125 KI mouse therefore is a model of recessive inheritance of the RTH.

Rod and cone photoreceptor cells of the retina are necessary for detection of light and color. TR- $\beta 2$ is expressed in the outer nuclear layer of the retina and regulates expression of $\mathrm{S}$ and $\mathrm{M}$ opsin in the cone photoreceptors, as demonstrated in TR- $\beta 2 \mathrm{KO}$ mice (8). In this study, TR- $\beta^{\mathrm{GS} / \mathrm{GS}}$ and TR- $\beta^{-/-}$mice displayed the same phenotype as TR- $\beta 2 \mathrm{KO}$ mice manifesting a selective loss of $\mathrm{M}$ opsin and concomitant increase in cone photoreceptors expressing $S$ opsin distributed in an anomalous pattern. Importantly, the total number of cone photoreceptors was not reduced in these models as compared with WT animals. These results indicate that opsin gene expression is regulated by TR- $\beta$.

A recent study, which compared thyroid hormone regulation of $\mathrm{S}$ - and $\mathrm{M}$-cone opsin enhancer elements, supports this conclusion (30). The S-cone opsin enhancer was activated by CRX, a photoreceptor-specific homeobox protein, and TR- $\beta 2$-inhibited gene expression from this enhancer. In contrast, the M-cone enhancer activity was not activated by either TR- $\beta 2$ or CRX; however, thyroid hormone treatment stimulated gene expression via this element. Thyroid hormone therefore has the potential to control opsin gene expression in the retina in much the same way as it affects myosin heavy chain $\alpha$ and $\beta$ (MHC- $\alpha$ and MHC- $\beta$ ) gene expression in the myocardium (31): stimulating one gene product (M-cone opsin and MHC- $\alpha$ ), while reciprocally inhibiting a related gene product (S-cone opsin and MHC- $\beta$ ).

The present analysis of hearing function in TR- $\beta^{-/-}$ mice is largely consistent with previous reports from the work of Forrest and colleagues with an 
independently derived mouse line. Both studies report an $A B R$ threshold elevation of approximately $60 \mathrm{~dB}(5,32)$. Importantly, the ABR in TR- $\beta \mathrm{GS} / \mathrm{GS}$ mice was not as severely affected as in TR- $\beta^{-/-}$mice, and at least at one frequency, hearing was nearly normal. The DPOAE data showed the same trends in cochlear sensitivity across the three genotypes. Hearing dysfunction was profound in TR- $\beta^{-/-}$mice, but TR- $\beta$ GS/GS mice were significantly less affected. Both lines display subtle anomalies in the structure of the tectorial membrane (Figure 5, g and h). Anomalies in tectorial membrane morphology are more pronounced when all TR isoforms are deleted (33), as well as when severe hypothyroidism is chemically induced during development (34).

The ABR represents summed activity of cochlear afferent fibers, which, in turn, constitute the final stage in the cochlea's transduction cascade from mechanical input (sound) into electrical output (neural spike trains). Thus, threshold elevation in the ABR could arise from dysfunction at many stages in cochlear processing. To better pinpoint the locus of dysfunction, the current study complemented the ABR measures with a DPOAE-based assay (Figure 5b). DPOAEs are sounds created by the inner ear, amplified by OHCs, and transmitted back to the ear canal, where they can be measured in the sound pressure waveform. As such, DPOAEs are a sensitive index of normal OHC function (25) and are not affected by destruction of IHCs and/or the cochlear neurons that innervate them. Thus, the virtually complete elimination of DPOAE responses in the TR- $\beta^{-/-}$mice demonstrates that the bulk of the threshold elevation in these mutants must originate in cochlear mechanics and/or micromechanics, i.e., the transformation of stapes motion to motion of the cochlear partition. There may also be changes in the electrical responses of IHCs and the process of synaptic transmission; indeed, previous workers have shown delays in the development of $\mathrm{K}$ channels in IHCs of these mutants (32). However, changes in IHC function cannot account for the profound DPOAE reductions.

A previous study by Forrest et al. (5) did not describe OHC loss as we observed in the TR- $\beta^{-/-}$ mice. As shown in Figure 5c, there was significant loss of OHCs in the basal third of the cochlea, especially for the TR- $\beta^{-/-}$mice. This loss was equally distributed among the three rows of OHCs. Significant hair cell loss was never seen in the WT ears. Similarly, among TR- $\beta^{\mathrm{GS} / \mathrm{GS}}$ mice, less pronounced and scattered OHC loss throughout the basal third of the cochlea was observed. The discrepancy between these studies may arise because the animals were older than those evaluated in the previous study, because of differences in the strain background, or because the hair cell loss is restricted to the "hook region" of the cochlear where the orientation of the epithelium causes it to be sectioned in atypical planes, thus complicating the analysis.
The large loss of OHCs and hearing capacity in TR- $\beta^{-/-}$versus TR- $\beta^{\mathrm{GS} / \mathrm{GS}}$ mice indicates that the action of TR in the inner ear might be different from in other tissues we examined. In the H-P-T axis and retina, these animals have an identical pathophysiologic phenotype. Clearly, TR DNA-binding is required for negative regulation of the H-P-T axis by thyroid hormone, calling into question models of thyroid hormone action that propose negative regulation of this axis in the absence of DNA-binding. Such models depend on the ability of non-DNAbound TR to sequester a limiting transcription factor in the absence (nuclear corepressor model) or presence (AP-1 model) of $\mathrm{T}_{3}$. In the former model, $\mathrm{T}_{3}$ treatment dissociates the corepressor and allows it to be recruited to the transcription complex (15); whereas in the latter model, $\mathrm{T}_{3}$ treatment blocks AP-1 activation by sequesting its coactivator protein(s) $(28,35)$. Regardless of their mechanism, however, these models cannot explain negative regulation of the H-P-T axis based on our findings.

Unexpectedly, however, TR-DNA binding was not absolutely required in the inner ear. Unlike TR- $\beta^{-/-}$ animals, TR- $\beta \mathrm{GS} / \mathrm{GS}$ mice displayed significant hearing ability and lacked significant OHC loss, suggesting that hearing development and/or maintenance can occur in the absence of TR DNA-binding. Although this could be due to some residual DNA-binding activity in the GS125 mutant, careful study of this mutation in vitro was unable to discern DNA-binding activity or function on any known TRE (17). Thus our findings indicate that, while TR- $\beta$ is essential for inner ear development, ear development is possible in the absence of TR- $\beta$ DNA-binding. It is suggested that other DNA-binding independent actions of TR may be important in vivo.

\section{Acknowledgments}

We would like to thank H. McDonalds for assistance with the immunohistochemistry studies, S. Radovick for advice in study design and interpretation, K. Ueda for technical advice, and A. F. Parlow of the NIH Pituitary Hormone Program, National Institute of Diabetes and Digestive and Kidney Diseases, for the TSH- $\beta$ antibody. This work was supported by NIH grants DC-0188 (to M.C. Liberman), DK 15070 (to S. Refetoff), and DK 50536 (to F.E. Wondisford).

\footnotetext{
1. Brent, G.A. 1994. The molecular basis of thyroid hormone action. N. Engl. J. Med. 331:847-853.

2. Oppenheimer, J.H., and Schwartz, H.L. 1997. Molecular basis of thyroid hormone-dependent brain development. Endocr. Rev. 18:462-475.

3. Koibuchi, N., and Chin, W.W. 2000. Thyroid hormone action and brain development. Trends Endocrinol. Metab. 11:123-128.

4. Cohen, R.C., Weintraub, B.D., and Wondisford, F.E. 2000. Chemistry and biosynthesis of thyrotropin. In Werner and Ingbar's The thyroid: a fundamental and clinical text. 8th edition. L.E. Braverman, and R.D. Utiger, editors. Williams \& Wilkins. Baltimore, Maryland, USA. 202-218.

5. Forrest, D., Erway, L.C., Ng, L., Altschuler, R., and Curran, T. 1996. Thyroid hormone receptor beta is essential for development of auditory function. Nat. Genet. 13:354-357.

6. Abel, E.D., et al. 1999. Divergent roles for thyroid hormone receptor
} 
beta isoforms in the endocrine axis and auditory system. J. Clin. Invest. 104:291-300.

7. $\mathrm{Ng}$, L., et al. 2001. A thyroid hormone receptor that is required for the development of green cone photoreceptors. Nat. Genet. 27:94-98.

8. Gauthier, K., et al. 1999. Different functions for the thyroid hormone receptors TRalpha and TRbeta in the control of thyroid hormone production and post-natal development. EMBOJ. 18:623-631.

9. Weiss, R.E., Forrest, D., Pohlenz, J., Cua, K., Curran, T., and Refetoff, S. 1997. Thyrotropin regulation by thyroid hormone in thyroid hormone receptor beta-deficient mice. Endocrinology. 138:3624-3629.

10. Abel, E.D., Ahima, R., Boer, M.-E., Elmquist, J.K., and Wondisford, F.E. 2001. Critical role for thyroid hormone receptor $\beta$ - 2 in the regulation of TRH neurons in the paraventricular hypothalamus. J. Clin. Invest. 107:1017-1023.

11. Bodenner, D.L., Mroczynski, M.A., Weintraub, B.D., Radovick, S., and Wondisford, F.E. 1991. A detailed functional and structural analysis of a major thyroid hormone inhibitory element in the human thyrotropin beta-subunit gene. J. Biol. Chem. 266:21666-21673.

12. Naar, A.M., et al. 1991. The orientation and spacing of core DNA-binding motifs dictate selective transcriptional responses to three nuclear receptors. Cell. 65:1267-1279.

13. Hollenberg, A.N., Monden, T., Flynn, T.R., Boers, M.E., Cohen, O., and Wondisford, F.E. 1995. The human thyrotropin-releasing hormone gene is regulated by thyroid hormone through two distinct classes of negative thyroid hormone response elements. Mol. Endocrinol. 9:540-550.

14. Sasaki, S., et al. 1999. Ligand-induced recruitment of a histone deacetylase in the negative-feedback regulation of the thyrotropin beta gene. EMBO J. 18:5389-5398.

15. Tagami, T., Madison, L.D., Nagaya, T., and Jameson, J.L. 1997. Nuclear receptor corepressors activate rather than suppress basal transcription of genes that are negatively regulated by thyroid hormone. Mol. Cell Biol. 17:2642-2648.

16. Reichardt, H.M., et al. 1998. DNA binding of the glucocorticoid receptor is not essential for survival. Cell. 93:531-541.

17. Shibusawa, N., Hollenberg, A.N., and Wondisford, F.E. 2003. Thyroid hormone receptor DNA binding is required for both positive and negative gene regulation. J. Biol. Chem. 278:732-738.

18. Bunting, M., Bernstein, K.E., Greer, J.M., Capecchi, M.R., and Thomas, K.R. 1999. Targeting genes for self-excision in the germ line. Genes Dev. 13:1524-1528

19. Williams, G.R. 2000. Cloning and characterization of two novel thyroid hormone receptor beta isoforms. Mol. Cell Biol. 20:8329-8342.

20. Sjoberg, M., Vennstrom, B., and Forrest, D. 1992. Thyroid hormone receptors in chick retinal development: differential expression of
mRNAs for alpha and N-terminal variant beta receptors. Development. 114:39-47.

21. Bradley, D.J., Towle, H.C., and Young, W.S., III. 1994. Alpha and beta thyroid hormone receptor (TR) gene expression during auditory neurogenesis: evidence for TR isoform-specific transcriptional regulation in vivo. Proc. Natl. Acad. Sci. U. S. A. 91:439-443.

22. Applebury, M.L., et al. 2000. The murine cone photoreceptor: a single cone type expresses both $\mathrm{S}$ and $\mathrm{M}$ opsins with retinal spatial patterning. Neuron. 27:513-523.

23. Dallos, P. 1992. The active cochlea. J. Neurosci. 12:4575-4585.

24. Brown, M.C., Nuttall, A.L., and Masta, R.I. 1983. Intracellular recordings from cochlear inner hair cells: effects of stimulation of the crossed olivocochlear efferents. Science. 222:69-72.

25. Liberman, M.C., et al. 2002. Prestin is required for electromotility of the outer hair cell and for the cochlear amplifier. Nature. 419:300-304.

26. Lazar, M.A. 1993. Thyroid hormone receptors: multiple forms, multiple possibilities. Endocr. Rev. 14:184-193.

27. Wondisford, F.E., Steinfelder, H.J., Nations, M., and Radovick, S. 1993. AP-1 antagonizes thyroid hormone receptor action on the thyrotropin b-subunit gene. J. Biol. Chem. 268:2749-2754.

28. Zhang, X.K., Willis, K.N., Husmann, M., Hermann, T., and Pfahl, M. 1991. Novel pathways for thyroid hormone receptor action through interaction with jun and fos oncogene activities. Mol. Cell Biol. 11:6016-6025.

29. Refetoff, S., Weiss, R.E., and Usala, S.J. 1993. The syndromes of resistance to thyroid hormone. Endocr. Rev. 14:348-399.

30. Yanagi, Y., Takezawa, S., and Kato, S. 2002. Distinct functions of photoreceptor cell-specific nuclear receptor, thyroid hormone receptor beta 2 and CRX in one photoreceptor development. Invest. Ophthalmol. Vis. Sci. 43:3489-3494.

31. Izumo, S., Nadal-Ginard, B., and Mahdavi, V. 1986. All members of the MHC multigene family respond to thyroid hormone in a highly tissuespecific manner. Science. 231:597-600.

32. Rusch, A., Erway, L.C., Oliver, D., Vennstrom, B., and Forrest, D. 1998. Thyroid hormone receptor beta-dependent expression of a potassium conductance in inner hair cells at the onset of hearing. Proc. Natl. Acad. Sci. U. S. A. 95:15758-15762.

33. Rusch, A., et al. 2001. Retardation of cochlear maturation and impaired hair cell function caused by deletion of all known thyroid hormone receptors. J. Neurosci. 21:9792-9800.

34. Deol, M.S. 1976. The role of thyroxine in the differentiation of the organ of Corti. Acta. Otolaryngol. 81:429-435.

35. Claret, F.-X., Antakly, T., Karin, M., and Saarcioglu, F. 1996. A shift in the ligand responsiveness of thyroid hormone receptor $\alpha$ induced by heterodimerization with retinoid $\mathrm{X}$ receptor $\alpha$. Mol. Cell Biol. 16:219-227. 